\title{
A case report of recurrent uterovaginal prolapse and cervical elongation in anulliparous female with a distorted Pelvis
}

\begin{abstract}
Recurrent uterovaginal prolapse adversely affects the life quality in women. Genitourinary prolapse affects around 40-50 \% of women in their lifetimes with a higher incidence in women of advanced age and parity. It is less common in females of reproductive age, being particularly rare in nulliparous females. The reported incidence in this group is only $1 \%$. In this case report we are highlighting a rare case of uterine prolapse in a young nulliparous woman after pelvic fracture. The cervix was also unusually long None of the previous surgeries took into account the elongated cervix with the prolapse, contributing to the socalled recurrence in this patient. The treatment of this patient was done by combining Sacro hysteropexy with Manchester Repair.
\end{abstract}

Keywords: recurrent uterovaginal prolapse, nulliparous, deformed pelvis, sacro hysteropexy
Volume 8 Issue 5 - 2020

Nazli Hameed, Binyamin Butt, Rabia Jamshaid
Obstetrics and Gynaecology, Shalamar Hospital Lahore, Pakistan

Correspondence: Nazli Hameed, Obstetrics and Gynaecology, Shalamar Hospital Lahore, Pakistan,

Email nazlihameed@gmail.com

Received: September 29, 2020 | Published: October 28, 2020

\section{Introduction}

Urogenital prolapsed adversely affects the quality of life of a woman especially in a younger age group. The prevalence rises with advanced age and rising parity. The trauma to the pelvic floor muscles and connective tissue after vaginal delivery contributes to this higher incidence. ${ }^{1}$ The condition is aggravated by hormonal deficiency after menopause, collagen density disorders, and factors contributing to increased intra abdominal pressure. ${ }^{1,2} \mathrm{~A}$ lower incidence is reported in younger nulliparous females without any predisposing weakness of supporting connective tissues. ${ }^{1-4}$ Restoration of sexual life and fertility concerns are the major issues in this age group. The re-operation rate is higher in this age group due to recurrence of symptoms after Manchester Repair in 3. 3\%-9. 5\% of the cases, improper surgical choice and poor surgical technique. A combination of these factors were operating in this particular case. She had a ventrisuspension procedure once and sacro hysteropexy second time to correct the prolapse, missing out the elongated cervix completely both on clinical examination and in advanced imaging investigations. The patient kept turning up for a presumed recurrence, because the elongated cervix with the covering cystocele had a remarkable similarity to the appearance of procidentia. Sacro hysteropexy combined with Manchester Repair, was hence chosen as a definitive treatment in this patient. ${ }^{3-6}$

\section{Case report}

A 30-year old nulliparous married for 2 years was admitted in Shalamar Hospital with complaint of something coming out of vagina for the last five years and urinary incontinence for last one year. She encountered an accident in the childhood and got multiple pelvic fractures. She had two failed surgeries for prolapse including ventri suspension and sacrohysteropexy in the last one year. Physical examination revealed displaced urethra and third degree uterovaginal prolapse. Cystogram confirmed cystocele formation during micturition and MRI Pelvis confirmed the diagnosis of third degree prolapse, deformed pelvis with malunited fracture of left ilium along sacroiliac joint. An unusually elongated cervix was not identified. Evaluation under anesthesia revealed an enlarged cervix of about 12 inches. Sacro hysteropexy was followed by cervical amputation and Manchester repair removing approximately 10 inches length of cervix. Postoperatively she recovered well. There has been no recurrence to this date.

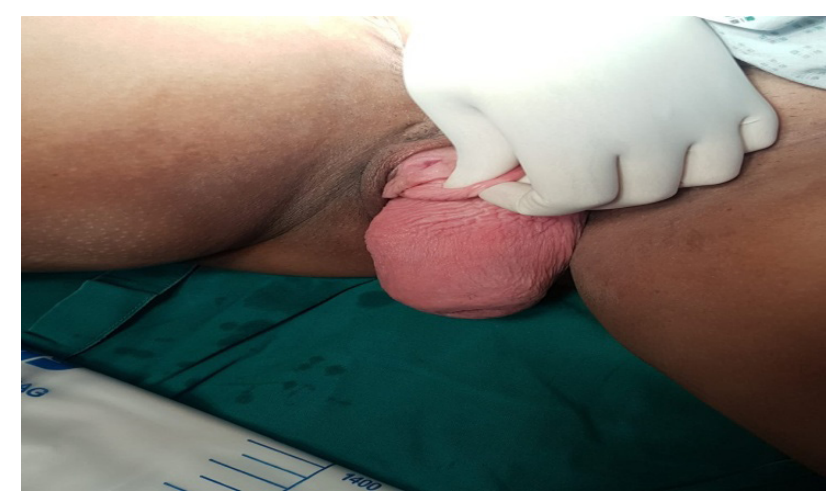

\section{Discussion}

Pelvic organ prolapse is the descent of pelvic viscera through the vaginal canal due to the weaker supporting structures of the pelvic floor. Surgery for this condition is one of the major planned gynecological surgeries. The risk of having surgery for pelvic organ prolapse is $11 \%$ with at least on third of the surgeries being for recurrence of prolapse. ${ }^{2,5}$ Almost half of women beyond the age of 50 years have varied degree of prolapse. The contributory factors besides increasing age are, childbirth trauma to pelvic floor muscles, factors causing raised intra abdominal pressure and Caucasian Race. The reported incidence of prolapse in nulliparous women is very low. ${ }^{5}$ Pelvic organ prolapse can involve three different compartments and are classified accordingly. Patient scan present with pressure symptoms, backache or something coming out of vagina. They may have lower urinary tract or bowel symptoms depending o the additional compartments involved. Any surgical intervention needs to 
be preceded by a thorough clinical evaluation of the patient including imaging techniques. Urodynamic studies are indicated in case of planned surgery for associated urinary symptoms. ${ }^{6-8}$ Optimization of general health and reduction of factors triggering prolapse should precede surgical management. Physiotherapy, pelvic floor exercises and intravaginal pessaries probably have a role in younger females with lesser degree of prolapse where fertility preservation gets a priority. Surgery offers definitive treatment. There are various surgical procedures for prolapse depending upon the type and degree of prolapse also considering the age of patient and fertility requirements. These include anteriorcolporrhaphy for cystourethocele, posterior colporrhaphy for rectocele, Manchester repair and Sacrohysteropexy. Pelvic fractures are also known to predispose to prolapse with a higher chance of recurrence. ${ }^{4-9}$ The resulting disfigurement and associated reduced sexual and urinary problems cause significant negative impact on patient's quality of life. ${ }^{5}$ The recurrence is due to a variety of factors including not only bony displacement like pubic diastasis but also associated injuries to the pelvic floor muscles, it's innervation, and connective tissue damage. Lap belt injuries have been reported to cause prolapse without even a direct hit to pelvic bones. ${ }^{8}$ The use of rectus abdominus muscle as a graft to bridge the defects has been reported. In vaginal hysterectomy the uterus is removed and gaps are repaired. ${ }^{10}$ Manchester Repair (Fothergill Repair) involves amputation of cervix and approximation of lateral ligaments on anterior aspect of cervical stump. Regarding the surgical care of this patient, a combination surgery was performed to address the recurrent prolapse and the unusually elongated cervix. There are very few reports describing the management of similar cases.
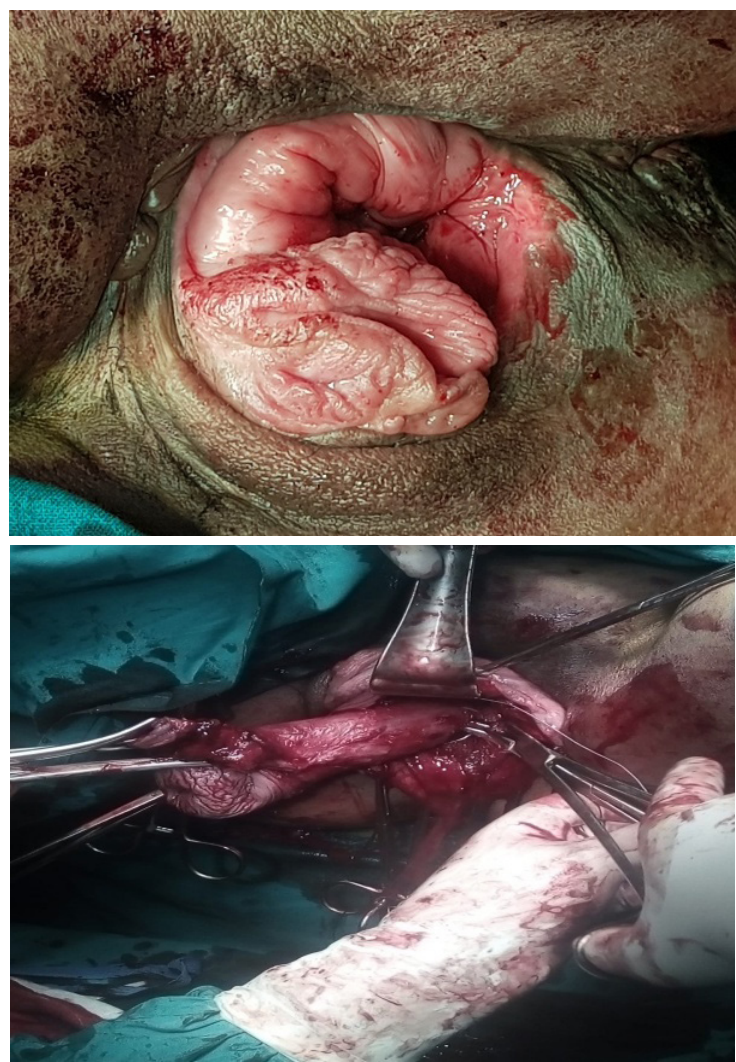

\section{Conclusion}

In conclusion, pelvic trauma is known but rarely observed cause of pelvic organ prolapse in nulliparous young women. Similarly an elongated cervix of this magnitude is a once in lifetime observation. This report highlights importance of a thorough pre-operative evaluation for getting a successful treatment outcome in for pelvic organ prolapsed where the normal pelvic anatomy is distorted due to a combination of different factors co existing.

\section{Consent}

Detailed consent was taken from patient regarding procedure and publication.

\section{Acknowledgments}

None.

\section{Funding}

None.

\section{Conflicts of interest}

Authors declares that there is no Conflict of interest.

\section{References}

1. Azinar AD, Hardianto G. Third Grade Uterine Prolapse in Young Age after Second Surgical Reconstruction. Obstet Gynecol Int J. 2015;2(4):00050.

2. American College of Obstetricians and Gynecologists. ACOG committee opinion no. 559: Cesarean delivery on maternal request. Obstet Gynecol. 2013;121:904.

3. Lakshmi Seshadri. Essentials of Gynaecology. 5th edition. Wolters Kluwer (India) Pvt. Ltd., New Delhi. 2014.

4. Ko P, Lo T. Delayed onset advanced pelvic organ prolapse after pelvic trauma in a nulliparous young female: case report. Int Urogynecol $J$. 2011;22(6):757-759.

5. Rajamaheswari N, Chhikara AB, Agarwal S. Vaginal mesh repair: is it appropriate for pelvic organ prolapse in a nulliparous female following pelvic ring fracture? Int Urogynecol J. 2013;24(7):1233-1236.

6. Mikos T, Papanicolaou A, Tsalikis T, et al. Uterine prolapse after pelvic trauma: case report and literature review. Int Urogynecol J. 2009;20:881884.

7. Ishida $\mathrm{H}$, Takahashi $\mathrm{K}$, Kurachi $\mathrm{H}$. Uterine prolapse during late pregnancy in a nulliparous woman. Int Urogynecol J. 2014;25(2):1739-1740.

8. Wright JL, Nathens AB, Rivara FP, et al. Specific fracture configurations predict sexual and excretory dysfunction in men and women 1 year after pelvic fracture. J Urol. 2006;176:1540-1545.

9. Handa VL, Blomquist JL, McDermott KC, et al. Pelvic floor disorders after vaginal birth: effect of episiotomy, perineal laceration, and operative birth. Obstet Gynecol. 2012;119(2 Pt 1):233.

10. Hefni M, El-Toucky T. Uterine prolapse in young women. Best Pract Res Clin Obstet Gynaecol. 2010;25(2):157-165. 